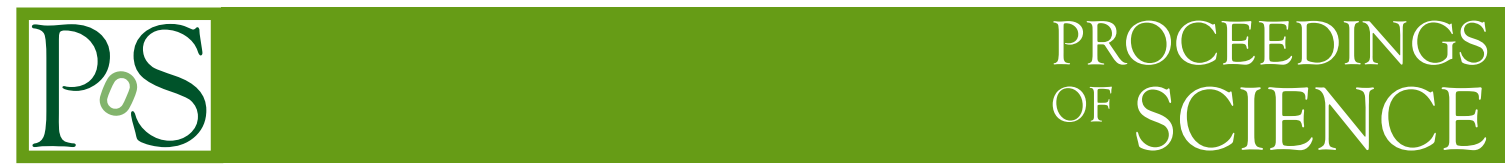

\title{
Searches for exotic Higgs boson decays at CMS
}

\author{
Nabarun Dev*t \\ University of Notre Dame (US) \\ E-mail: ndevend.edu
}

\begin{abstract}
A summary of recent results of direct searches for exotic decays of the $125 \mathrm{GeV}$ Higgs boson is presented. The searches presented include lepton flavor violating (LFV) Higgs decay searches followed by searches for invisible Higgs decays. These searches were done with data collected by the CMS detector at the LHC in 2015 and 2016 at a center-of-mass energy of $13 \mathrm{TeV}$.
\end{abstract}

The European Physical Society Conference on High Energy Physics

5-12 July

Venice, Italy

* Speaker.

${ }^{\dagger}$ On behalf of the CMS collaboration. 


\section{Introduction}

The standard model (SM) of particle physics is a well-tested description of nature. After the discovery of a Higgs Boson in 2012 by the CMS and ATLAS experiments [1, 2], one of the most important tasks for particle physicists is to assess its compatibility with the SM Higgs boson. Many results obtained so far have indicated that it has properties such as spin, couplings, CP that are compatible with the SM Higgs. However, analysis of Higgs couplings by CMS and ATLAS have set an upper limit at 95\% confidence level (CL) on beyond standard model (BSM) Higgs decays of $34 \%$ [3], leaving large room for searches targeting exotic decays. These decays could provide a portal for us to study the BSM Higgs sector.

The CMS collaboration has been broadening the coverage of exotic decay searches in RUN 2 and this document briefly describes recent results obtained. The CMS detector is described in detail in [4].

\section{Lepton flavor violating decays of Higgs (125)}

LFV decays of the Higgs are forbidden in the SM but are allowed by several BSM models such as Randall-Sundrum, composite Higgs and supersymmetric models. Direct searches for $H \rightarrow \mu \tau$ and $H \rightarrow e \tau$ with $35.9 \mathrm{fb}^{-1}$ data collected by CMS at $13 \mathrm{TeV}$ center-of-mass energy have been performed [5]. Both hadronic and leptonic decays of the tau leptons were considered. Final states with two same-flavor leptons were not studied due to large Drell-Yan background.

Events were divided into four categories for signal extraction. These categories enhance different Higgs production modes and are based on number of jets in the event with $p_{T}>30 \mathrm{GeV}$ and $|\eta|<4$.7. The 0 -jet category targets ggH contribution while the 1 -jet category targets ggH production with initial state radiation (ISR). The 2-jet category is further divided into two categories based on the invariant mass of the two jets $\left(M_{j j}\right)$. The category with high $M_{j j}$ targets VBF events and the other targets ggH events with several jets from ISR.

The primary backgrounds for this search come from Drell-Yan production which is the dominant background in 0 -jet category and $t \bar{t}$ production which is a dominant background in 2 -jet category. 1-jet category is dominated by Drell-Yan for hadronic and $t \bar{t}$ for fully leptonic channels. Both these backgrounds are estimated from simulations. The reducible background (where a jet is misidentified as a lepton) comes predominantly from $\mathrm{W}+$ jets and QCD events. It is estimated using fully (partially) data-driven techniques in hadronic (fully leptonic) channels where it is larger (smaller). All other smaller backgrounds such as diboson, single top quark, $\mathrm{W} \gamma$ and SM Higgs production are estimated using simulation. Simulated backgrounds are corrected with several scale factors to account for the difference in selection efficiencies between data and simulation.

The $\tau$ being much lighter than the Higgs, the missing transverse momentum (MET) is closely aligned to the $\tau$ decay products giving us a way to separate signal from background. This also motivates us to use the collinear approximation [6] to form an estimator of the Higgs mass, called the collinear mass, where the $p_{T}$ of the neutrinos is estimated from the projection of the MET on the visible $\tau$ decay product direction. Also, leptons from signal processes are expected to have a generally harder $p_{T}$ spectrum compared to the background. This helps in reduction of backgrounds 
like Drell-Yan and SM Higgs. Further, the invariant mass formed from transverse components of one of the leptons and the MET is also used to achieve discrimination of signal and background.

A boosted decision tree using the above variables as inputs is trained with signal against a combination of backgrounds for each of the channels. The results are extracted using a binned likelihood fit over the BDT output distributions. (A cut-based analysis using collinear mass as signal variable was kept as a cross-check and was found to produce compatible albeit less sensitive results). No significant excess of data was observed over the background. The $C L_{s}$ method [7] was used to set upper limits on $H \rightarrow \mu \tau$ and $H \rightarrow e \tau$ branching fractions at $95 \%$ confidence level (figure 1). The observed (expected) upper limits are $0.25 \%(0.25 \%)$ and $0.63 \%(0.37 \%)$ for $B R(H \rightarrow \mu \tau)$ and $B R(H \rightarrow e \tau)$ respectively while the best fit branching fractions are $0.00 \pm 0.12$ and $0.30 \pm 0.18$.
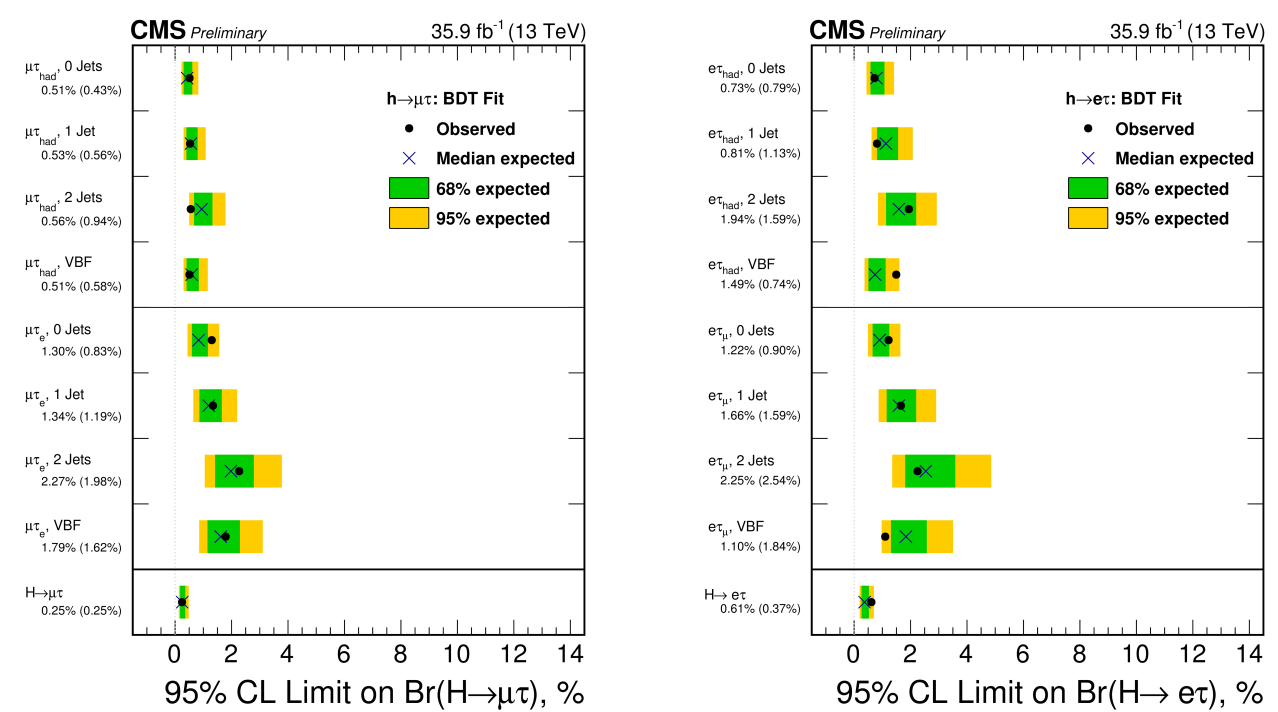

Figure 1: Observed (Expected) 95\% CL upper limits on BR $(H \rightarrow \mu \tau)$ (left) and BR $(H \rightarrow e \tau)$ (right) [5].

The constraints on these branching fractions can be interpreted in terms of LFV Yukawa couplings. The 95\% CL limit on these couplings assuming a SM Higgs decay width of $4.1 \mathrm{MeV}$ and mass of $125 \mathrm{GeV}$ are given in Table 1 .

\begin{tabular}{ccc}
\hline Channel & Coupling & 95\% CL limit \\
\hline$H \rightarrow \mu \tau$ & $\sqrt{\left|Y_{\mu \tau}\right|^{2}+\left|Y_{\tau \mu}\right|^{2}}$ & 0.0014 \\
\hline$H \rightarrow e \tau$ & $\sqrt{\left|Y_{e \tau}\right|^{2}+\left|Y_{\tau e}\right|^{2}}$ & 0.0023 \\
\hline
\end{tabular}

Table 1: 95\% CL observed upper limit on the LFV Yukawa couplings.

These LFV Higgs results are the most sensitive till date and exclude a previous $2.4 \sigma$ excess observed by CMS in the $H \rightarrow \mu \tau$ channel [8].

\section{Invisible decays of the Higgs (125)}

Several BSM models allow Higgs decays into invisible particles. These include supersymmet- 
ric models and large extra dimensional models. Several searches are performed targeting different production modes. The characteristic signature of all such searches is large MET from the invisible decay products recoiling against visible particles.

\section{1 $Z \rightarrow l l+$ MET}

This search targets the $\mathrm{ZH}$ production mode of the Higgs with the $\mathrm{Z}$ subsequently decaying into electron (muon) and anti-electron (anti-muon). It was performed using $35.9 \mathrm{fb}^{-1}$ data collected by the CMS at $13 \mathrm{TeV}$ center-of-mass energy [9]. Two well-isolated leptons of opposite sign and same flavor are required to be present in the event along with large MET. The invariant mass of the dilepton pair is required to be compatible with the $\mathrm{Z}$ boson mass within a tight (15 $\mathrm{GeV})$ window. The dominant backgrounds in this search are $Z Z(2 l 2 v)$ production and $W Z(3 l 2 v)$ production where one lepton misses identification. A binned max likelihood fit to the MET shape was used to extract results. The sensitivity of the search was further improved by about $10 \%$ by training a boosted decision tree to discriminate signal from background and using its output shape as a template for the fit. No excess of events was observed over the background prediction. Observed (expected) upper limits of $40 \%(42 \%)$ were set at 95\% CL on branching fraction of Higgs decaying into invisible particles.

\subsection{One or two jets recoiling against MET}

This search targets events with one or two jets recoiling against large MET which is the signature of $\mathrm{Z}(\mathrm{W}) \mathrm{H}$ production with $\mathrm{Z}(\mathrm{W})$ decaying hadronically or of ggF production in association with ISR. The main backgrounds come from $Z(v v)+$ jets and $W(l v)+$ jets production. Events are divided into two categories for signal extraction, one with high $p_{T}$ fat jets in the event and another without. A simultaneous fit is performed of MET distributions in the signal region and several control regions to extract results. No excess of events was seen and an observed (expected) upper limit of 53\% (40\%) was set on branching fraction of Higgs to invisible decays at 95\% CL. $35.9 \mathrm{fb}^{-1}$ data collected by the CMS at $13 \mathrm{TeV}$ center-of-mass energy in 2016 was used to extract these limits [10].

\subsection{VBF Higgs invisible search}

Events in which a Higgs boson is produced via vector boson fusion is targeted by this search. Two jets with high rapidity gap and high dijet invariant mass are required to be present along with large MET. A dedicated trigger was used to select such events. Major backgrounds are estimated using data-driven techniques using control regions with one or more leptons. The QCD background is reduced by requiring the jets to recoil against MET. This search was performed with $2.8 \mathrm{fb}^{-1}$ of data collected by CMS in 2015 [11]. Observed (expected) upper limits of 69\% (62\%) was set at 95\% CL on the branching fraction Higgs decaying to invisible particles.

\subsection{Combination of Higgs invisible searches}

Finally, a combination of several searches targeting different production modes of Higgs with $4.9 \mathrm{fb}^{-1}, 19.7 \mathrm{fb}^{-1}$ and $2.3 \mathrm{fb}^{-1}$ data collected at 7, 8 and $13 \mathrm{TeV}$ center-of-mass energy respectively was performed [12]. Observed (expected) upper limits at 95\% confidence level on branching fraction of Higgs boson decaying to invisible particles was set at 24\% (23\%). 


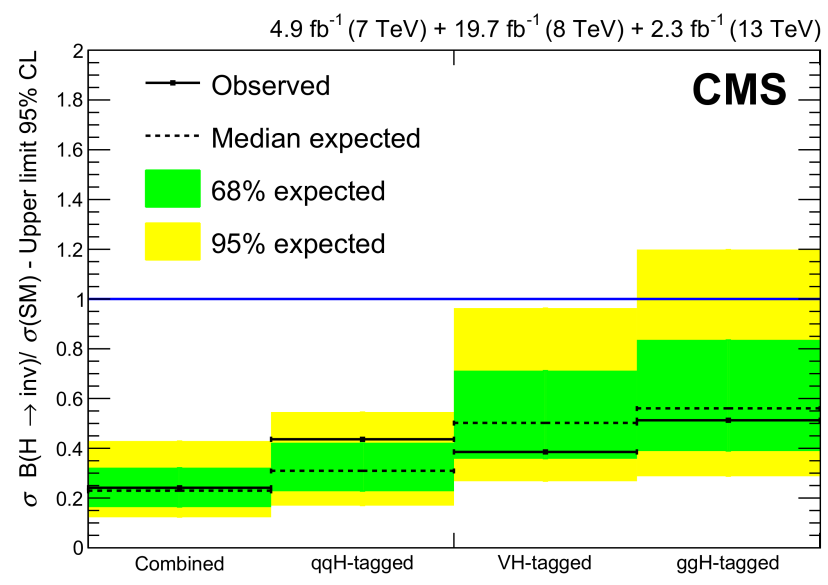

Figure 2: Observed (Expected) 95\% CL upper limits on BR $(H \rightarrow$ invisible $)$ decays from a combination of several searches [12].

\section{Conclusion}

SM Higgs provides a portal to probe physics beyond the standard model. Exotic Higgs decays would bring direct evidence of such new physics. Recent results of such searches by the CMS collaboration has been presented. No excess of data over background prediction was observed in any of the searches discussed. Stringent limits on branching fraction have been set on lepton flavor violating decays of the Higgs and Higgs to invisible decays. Other such analyses are also being performed with the $13 \mathrm{TeV}$ dataset, and with more data being collected, CMS hopes to increase the sensitivity of Higgs exotic decay searches in the near future.

\section{References}

[1] CMS Collaboration, Observation of a new boson at a mass of $125 \mathrm{GeV}$ with the CMS experiment at the LHC, Phys. Lett. B 716 (2012) 30 [ arXiv:1207.7235]

[2] ATLAS Collaboration, Observation of a new particle in the search for the Standard Model Higgs boson with the ATLAS detector at the LHC, Phys. Lett. B 716 (2012) 1 [ arXiv:1207.7214]

[3] ATLAS, CMS Collaborations, Measurements of the Higgs boson production and decay rates and constraints on its couplings from a combined ATLAS and CMS analysis of the LHC pp collision data at $\sqrt{s}=7$ and $8 \mathrm{TeV}$, JHEP 08 (2016) 045 [arXiv:1606.02266]

[4] CMS Collaboration, The CMS experiment at the CERN LHC, JINST 3 (2008) S08004

[5] CMS Collaboration, CMS-PAS-HIG-17-001, (2017) [https://cds.cern.ch/record/2273176]

[6] R. K. Ellis, I. Hinchliffe, M. Soldate, and J. van der Bij, Higgs Decay to tau+ tau-: A Possible Signature of Intermediate Mass Higgs Bosons at the SSC, Nucl. Phys. B297 (1988) 221[ doi:10.1016/0550-3213(88)90019-3.]

[7] A. L. Read, Presentation of search results: the $C L_{s}$ technique, J. Phys. G 28 (2012) 2693 [ doi:10.1088/0954-3899/28/10/313] 
[8] CMS Collaboration, Search for lepton-flavour-violating decays of the Higgs boson, Phys. Lett. B 749 (2015) 337 [arXiv:1502.07400]

[9] CMS Collaboration, CMS-PAS-EXO-16-052, (2017) [https://cds.cern.ch/record/2278344]

[10] CMS Collaboration, CMS-PAS-EXO-16-048, (2017) [https://cds.cern.ch/record/2280270]

[11] CMS Collaboration, CMS-PAS-HIG-16-009, (2017) [https://cds.cern.ch/record/2142460]

[12] CMS Collaboration, Searches for invisible decays of the Higgs boson in pp collisions at $\sqrt{s}=7,8$ and $13 \mathrm{TeV}$, JHEP 1702 (2017) 135 [ arXiv:1610.09218] 